\title{
Diabetes and depression in Lebanon and association with glycemic control: a cross-sectional study
}

This article was published in the following Dove Press journal: Diabetes, Metabolic Syndrome and Obesity:Targets and Therapy

\author{
Hala Ahmadieh* \\ Hadi Itani* \\ Sanaa Itani \\ Khaled Sidani \\ Mona Kassem \\ Kassem Farhat \\ Mohammad Jbeily \\ Abdallah Itani
}

Department of Internal Medicine, Faculty of Medicine, Beirut Arab University, Beirut, Lebanon

*These authors contributed equally to this work

Correspondence: Hala Ahmadieh Faculty of Medicine, Beirut Arab University, PO Box: II-5020

Riad El Solh, I I072809, Beirut, Lebanon

Tel +961 70209135

Email hala.ahmadieh@bau.edu.lb
Background: Diabetes mellitus is a chronic noncommunicable disease characterized by hyperglycemia and is associated with chronic complications affecting the overall quality of life. As of 2017, the prevalence of diabetes in Lebanon is estimated to be $14.6 \%$. Depression is noted to be common among Lebanese citizens, present in around $17.3 \%$. This study aims to investigate the prevalence of depression among the diabetic Lebanese citizens and to study its relationship with poor glycemic control and diabetes complications.

Methods: In total, 436 diabetic patients participated in this cross-sectional study. Patients with diabetes mellitus attending several private clinics and health care centers were asked to fill out a well-structured questionnaire developed by an expert. Depression was assessed using Beck Depression Inventory (BDI). The study collected demographic information about the participants including their lifestyles, their last reported glycosylated hemoglobin values, and their reported microvascular and macrovascular complications. Data were collected, entered, and analyzed on SPSS software version 23.1. A descriptive analysis is carried out by calculating the mean and standard deviation for continuous variables and number and percentage for categorical ones. Association between categorical variables is evaluated using a chi-squared test. A $P$-value of $<0.05$ is considered to be significant.

Results: Depression was prevalent among $28.8 \%$ of the patients with diabetes mellitus. The average age of participants was 64.08 years. In total, $280(64.2 \%)$ of the participants were females and the average diabetes duration was 8.89 years. There was no significant relationship found between depression and glycemic control. By contrast, a significant association between retinopathy, nephropathy, and stroke and depression was found.

Conclusion: Depression was found to be present among $28.8 \%$ of the patients with diabetes mellitus in Lebanon; however, no association was established between depression and glycemic control. Keywords: glycemic control, quality of life, complications, glycosylated hemoglobin, diabetes, depression

\section{Plain language summary}

Diabetes mellitus, being highly prevalent, is characterized by high levels of blood sugar and is often associated with long-term complications affecting the retina of the eye, the nerves, and the kidneys, and may lead to higher risk of cardiac diseases or strokes. Depression is a mental illness which leads to symptoms such as low mood, not getting any pleasure from doing daily activities, and having weight and sleep changes, in addition to other symptoms. This study tries to find if an association exists between diabetes mellitus and depression and between diabetes control and depression. Participants with diabetes mellitus were asked to fill a survey and information was obtained about their age, gender, diabetes duration, diabetes 
control, and whether they got any complications associated with diabetes mellitus. In addition, a well-validated scale was used to assess whether they got depression or not and the severity of their depression. It was found that $29 \%$ of patients with diabetes mellitus in our study had depression. No association was found between diabetes control and depression; however, an association was found between certain complications associated with diabetes and depression.

\section{Introduction}

Diabetes mellitus (DM) is a chronic debilitating disease that affects a huge number of people, disturbing their quality of life. ${ }^{1}$ It is a widespread disease; the International Diabetes Federation (IDF) estimated that 425 million people were diagnosed with DM in 2017. This number is expected to increase up to 629 million by 2045 with the majority of cases occurring in low- and middle-income countries. ${ }^{2}$ It was found that from the 425 million patients worldwide, an average of 38.7 million resided in the Middle East and North Africa, ${ }^{3}$ with an average of 585,400 living in Lebanon. ${ }^{4}$ The increase in prevalence of DM in the Arab world can be a result of many factors including obesity, sedentary life style, and genetic predisposition. ${ }^{5}$

DM causes microvascular complications such as diabetic nephropathy, retinopathy, and neuropathy and macrovascular complications such as coronary artery disease, stroke, and peripheral artery disease. ${ }^{6}$ These complications may lead to psychological disturbances, including depression. ${ }^{7}$ A meta-analysis done among 42 published studies that included 21,351 adults found that the prevalence of major depression and clinically relevant depression in diabetic patients was $11 \%$ and $31 \%$, respectively. ${ }^{8}$ According to American Psychiatric Association, depression is a serious medical illness that negatively affects how a person feels, thinks, and acts. ${ }^{9}$ In Lebanon, the prevalence of depression was estimated to be $17.3 \%$ among its population. ${ }^{10}$

Glycemic control is usually monitored using glycosylated hemoglobin (HbA1c) level, which is considered the most reliable test to follow the disease's progression, assess the patient's response to treatment, and monitor the patient's glycemic control in the past 2-3 months. ${ }^{11,12}$ The American Diabetes Association (ADA) recommends that the ideal rate of $\mathrm{HbA1c}$ for nonpregnant diabetic adults must be less than $7 \% .{ }^{13}$ Diabetic patients with poor glycemic control, having higher levels of $\mathrm{HbAlc}$, are found to be more depressed and anxious than patients who had good glycemic control, ${ }^{14,15}$ regardless of the presence of many confounders. ${ }^{16}$ In Peru, the prevalence of depression in type $2 \mathrm{DM}$ is high compared with the population, and patients with depression had a 1.3 times greater prevalence of poor glycemic control ( $\mathrm{HbA} 1 \mathrm{C} \geq 7) .{ }^{16}$ Furthermore, a study conducted by Carreira et al on type $1 \mathrm{DM}$ found the prevalence of depression to be $21.7 \%$ and associated variables included female gender, unemployment, smoking, diabetic complications, lack of support, high number of weekly hyperglycemia, and poor quality of life. ${ }^{17}$ Camara et al noticed that depressive symptoms are worse in patients with $\mathrm{HbA} 1 \mathrm{C}$ value $>9 \%{ }^{18}$

Studies showed that decrease in HbA1C level is associated with a decreased risk of diabetic complications, whether microvascular or macrovascular. According to the United Kingdom Prospective Diabetes Study (UKPDS), a 1\% decrease in $\mathrm{HbA} 1 \mathrm{C}$ value results in $37 \%$ decrease in risk of microvascular complications and $21 \%$ of other type 2 DMrelated diseases. ${ }^{19}$ The Diabetes Control and Complications Trial/Epidemiology of Diabetes Interventions and Complications (DCCT/EDIC) study demonstrated a 35\%-76\% reduction in the early stages of microvascular complications, with a median $\mathrm{HbA} 1 \mathrm{c}$ of $7 \%$ in type 1 diabetic patients. ${ }^{20}$ Therefore, diabetic patients must maintain lifelong medical attention in order to decrease the occurrence of diabetic complications to improve the quality of life that can affect clinical outcomes of the disease. ${ }^{21}$

Among the depressed diabetic patients, there are several factors that increase depression level other than DM itself: low income and poor socioeconomic status are among the principal ones. ${ }^{12,22,23}$ In addition, patients with supportive and continuous medical care display less depression. ${ }^{24}$ Also, age and form of medication determine the level of depression. ${ }^{25}$ Berge et al concluded that diabetic patients in their 40s had twice more depression than nondiabetic patients, and patients in their 40s receiving oral treatment had a three times higher depression prevalence. ${ }^{25}$ Another determinant of depression is the gender. Katon stated that female diabetics were more likely to be affected by depression (28\%) than diabetic males were $(18 \%)^{26}$

Depression can affect patients with DM in many ways. Patients with depression and DM have poor adherence to diet and have trouble taking the medications as prescribed which leads to severe hypoglycemic episodes. ${ }^{27}$ Depression in patients with DM is usually not diagnosed early and it is not treated until 6 months after diagnosis. ${ }^{28,29}$ There is a bidirectional relation existing between DM and depression: having DM increases the risk of developing depression and the opposite is also possible. ${ }^{30}$ 
Since to the best of our knowledge no data are available on the subject, we propose to: determine the prevalence of depression among the diabetic patients in Lebanon; investigate whether DM complications and poor glycemic control are related to a higher prevalence of depression in these patients; and evaluate the association of demographic, social, and therapeutic factors with the level of depression among patients with diabetes.

\section{Materials and methods Study design and settings}

This is a descriptive cross-sectional study that was carried out between April 2017 and April 2018. Data were collected between June 2017 and September 2017. The study was conducted in Lebanon by interviewing patients in public, several private clinics, and health care centers across Lebanese territories.

Ethical approval was obtained from the directors of the health care centers by presenting a formal request from Beirut Arab University Institutional Review Board (BAU IRB) (Approval code: 2017 H-0057-M-R-0225).

\section{Sampling and sample size}

Patients were selected by convenient sampling from all Lebanese governorates. A minimum of 384 participants were selected, with no limit in the maximum number of participants. The minimum sample size was calculated based on a confidence level of $95 \%$ and margin of error of $5 \%$ with population size estimated to be 6 million. These measures were selected to ensure that enough representation can be achieved to test different hypotheses for further analysis of the results. A pilot study was performed on 20 nondiabetic volunteers of different age groups and socioeconomic states to evaluate the procedure of assessment and the randomization of the research, and the questionnaire was edited based on its results to be suitable for a larger study population.

\section{Inclusion and exclusion criteria}

The study included people residing in Lebanon of all nationalities who are diagnosed with either type 1 or type 2 DM, whether treated or not.

Eligible candidates should be aged 16 and above, male or female, capable of answering the questions to complete the questionnaire and willing to take part in this study. BAU IRB approved that all participants, including those aged between 16 and 18, can provide written informed consent prior to their participation. All regions of Lebanon were included. Excluded patients were those with mental and cognitive impairment who were unable to understand or to answer the questions, those who suffered from emotional drives while proceeding with the questionnaire including those who started crying and those who felt unwell when they were asked certain questions because of personal problems, and pregnant women.

\section{Variables of interest and criteria of depression}

A questionnaire was designed by a team of experts; each one took around 15 minutes to be completed. It was divided into four sections. The first section included information about the patient's age, gender, marital status, employment status, socioeconomic status, and governorate of residency. The second contained questions about the patient's exercise habits, smoking habits, and sleeping hours. The third one comprised information about medical follow-up in terms of diabetic years, $\mathrm{HbA} 1 \mathrm{C}$ value, medication, commitment to treatment, treatment bothersome, associated complications, and source of awareness about DM, diet annoyance, physician visits, hospital admissions, physician-patient relation, and disease perception. In the last section, the exposure of interest was depression, and Beck Depression Inventory (BDI) was used to assess it.

BDI is a set of 21 self-reported items that assess symptoms and attitude of depression. Each item had an intensity ranging between 0 and $3 .{ }^{31}$ It is a globally used, validated scale that requires few minutes to be completed. ${ }^{32}$ Arabic version of the scale was also included. It was back-toback translated and validated by a legal translator (Professor Abdelsattar Ibrahim). ${ }^{33}$ The cutoff of 16 was used to detect depression such as individuals with scores $>16$ were considered depressed, and scores $\leq 16$ were considered normal. ${ }^{34}$ The cutoff of 16 showed the best balance between sensitivity and predictive values among diabetic patients. ${ }^{34}$ Depression was further categorized into four levels: scores between 17 and 20 were considered to be borderline clinical depression; 21-30 were moderately depressed; 31-40 were severely depressed, and above 40 were extremely depressed. ${ }^{35}$

Patients were asked to provide their recent $\mathrm{HbA1C}$ data to know objectively what their recent glycemic control have been, instead of relying on information obtained from the patients' memories. According to ADA, a cutoff of seven was adopted to determine glycemic control, where values $\geq 7$ indicated poor glycemic control and values $<7$ indicated good glycemic control. ${ }^{13}$ Regarding diabetic complications, 
they were self-reported, and participants were asked to choose from a provided list of diabetic complications included in the questionnaire.

\section{Statistical analysis}

Data were entered into the Statistical Package of Social Science (SPSS version 23.1; IBM Corporation, Armonk, NY, USA), which was then used for data reviewing (data were double checked), management, and analyses using built range checks with automatic error prompts. In addition, ascending/descending order view of variables and descriptive histograms were performed to easily identify outliers, but none were found.

Analyses were carried out by calculating the mean and standard deviation for continuous variables and number and percentage for categorical ones. Continuous variables included the HbAlc value of each participant and the BDI score. Categorical variables included age, gender, place of residence, occupational status, marital status, nationality, monthly income, sports practice, smoking, daily sleeping hours, duration of diabetes since diagnosis, recent $\mathrm{HbA1c}$ testing (past 6 months), HbA1c value categories (good vs bad glycemic control), form of medication, types of oral drugs, commitment to treatment, satisfaction with treatment, refusal of insulin intake, causes of insulin refusal, duration of insulin therapy, number of visits to the physician (in the past 6 months), number of admissions to hospitals (in the past 6 months), patient relief by the physician, sources of patient education about DM, DM complications, bothersome from DM complications, presence of hypoglycemic symptoms, frequency of occurrence of hypoglycemic episodes, annoyance from having diet restrictions, social performance impairment due to DM, self-perceived health status, self-reported disease perception, and levels of depression.

Throughout the study, chi-squared, ANOVA, $t$-test, as well as exploratory tests were used.

\section{Results}

\section{Prevalence of depression}

From the 450 subjects initially approached to participate, 440 accepted but four were excluded due to missing data or refusal to continue, hence a total of 436 diabetic patients were included in this study. In total, 125 patients (28.8\%) were found to have depression. The mean BDI score was found to be 13.45 (SD: 9.36) among all participants. Depressed patients had a mean BDI score of 25.65, while nondepressed patients had a mean of 8.54. Among the depressed patients, 36 had borderline clinical depression $(28.8 \%)$, 59 had mod- erate depression (47.2\%), 25 had severe depression $(20 \%)$, and only five had extreme depression (4\%).

\section{Population characteristics}

The average age of the participants was 64.08 years (SD: 17.06). In total, 280 were females $(64.2 \%)$, with average disease duration of 8.89 years (SD: 5.59 ).

Table 1 shows the different frequencies of levels of depression among population characteristics.

Further exploratory studies and ANOVA for the significantly associated variables were done to determine which subcategories were associated with higher BDI scores. Results showed that living in Beqaa and being retired had higher means with scores being 16 (SD: 9.16) and 17.98 (SD: 10.09), respectively.

In total, $279(64 \%)$ of the participants were married, 47 were single (10.8\%), 14 were divorced $(3.2 \%)$, and $96(22 \%)$ were widowed. There was no significant relationship between marital status and depression $(P=0.401)$.

When asked about sports practice, 173 (39.7\%) participants revealed practicing sports. Sports practice, however, had no significant relation to depression $(P=0.337)$.

\section{Diabetes and disease management}

Moving on to DM-related issues, patients were asked if they had measured their HbA1c levels in the past 6 months. In total, $356(81.5 \%)$ presented their recent HbA1c levels during the time of data collection. The regular measurement of HbA1c blood levels was significantly associated with depression $(P=0.031)$; those who measured their HbAlc levels in the past 6 months had a mean BDI score of 13.23 (SD: 8.96), whereas those who did not had a mean score of 14.59 (SD: 12.09).

The mean HbA1c was 7.9 (SD: 1.95). A cutoff of 7 was used to determine the glycemic control, and it was found that $156(43.8 \%)$ had it below or equal to 7 while $200(56.2 \%)$ had it above 7 . There was no significant correlation between glycemic control and depression $(P=0.843)$.

Table 2 shows the percentages of participants with either good or poor glycemic control among different levels of depression.

Upon questioning about treatment, 429 (98.39\%) were being treated.

Table 3 shows the frequencies of different levels of depression among different forms of medication, types of oral drugs used, and insulin therapy duration.

When asked about recommendations for insulin therapy, 49 (11.2\%) of all participants admitted refusing 
Table I Characteristics of the study population according to different levels of depression

\begin{tabular}{|c|c|c|c|c|c|c|}
\hline & \multicolumn{6}{|c|}{ Depression levels } \\
\hline & Nondepressed & $\begin{array}{l}\text { Borderline clinical } \\
\text { depression }\end{array}$ & $\begin{array}{l}\text { Moderate } \\
\text { depression }\end{array}$ & $\begin{array}{l}\text { Severe } \\
\text { depression }\end{array}$ & $\begin{array}{l}\text { Extreme } \\
\text { depression }\end{array}$ & $P$-value ${ }^{a}$ \\
\hline \multicolumn{7}{|l|}{ Place of residence } \\
\hline Beirut & $156(50.2 \%)$ & $24(66.7 \%)$ & 43 (72.9\%) & 17 (68\%) & $4(80 \%)$ & \\
\hline South & $99(31.8 \%)$ & $5(13.9 \%)$ & $4(6.8 \%)$ & I (4\%) & 0 & \\
\hline North & $20(6.4 \%)$ & I (2.8\%) & $5(8.5 \%)$ & $2(8 \%)$ & I (20\%) & 0.007 \\
\hline Beqaa & $5(1.6 \%)$ & I (2.8\%) & $\mathrm{I}(\mathrm{I} .7 \%)$ & I (4\%) & 0 & \\
\hline Mount Lebanon & $31(10 \%)$ & $5(13.9 \%)$ & $6(10.2 \%)$ & $4(16 \%)$ & 0 & \\
\hline \multicolumn{7}{|l|}{ Gender } \\
\hline Male & $106(34.1 \%)$ & 15 (4I.7\%) & $25(42.4 \%)$ & $8(32 \%)$ & $2(40 \%)$ & 0691 \\
\hline Female & 205 (65.9\%) & 21 (58.3\%) & $34(57.6 \%)$ & $17(68 \%)$ & $3(60 \%)$ & 0.691 \\
\hline \multicolumn{7}{|l|}{ Nationality } \\
\hline Lebanese & $211(67.8 \%)$ & 27 (75\%) & 48 (8I.4\%) & $14(56 \%)$ & $3(60 \%)$ & \\
\hline Syrian & $40(12.9 \%)$ & 4 (11.1\%) & $6(10.2 \%)$ & $8(32 \%)$ & $2(40 \%)$ & 0126 \\
\hline Palestinian & $59(19 \%)$ & $4(11.1 \%)$ & $5(8.5 \%)$ & $3(12 \%)$ & 0 & 0.126 \\
\hline Others & $\mathrm{I}(0.3 \%)$ & I (2.8\%) & 0 & 0 & 0 & \\
\hline \multicolumn{7}{|l|}{ Occupation } \\
\hline Employed & $80(25.7 \%)$ & $5(13.9 \%)$ & II (I8.6\%) & $5(20 \%)$ & 0 & \\
\hline Unemployed & $176(56.6 \%)$ & $21(58.3 \%)$ & 31 (52.5\%) & $9(36 \%)$ & $2(40 \%)$ & \\
\hline Student & $4(1.3 \%)$ & I (2.8\%) & 0 & I (4\%) & 0 & 0.037 \\
\hline Retired & $51(16.4 \%)$ & $9(25 \%)$ & $18(28.8 \%)$ & $10(40 \%)$ & $3(60 \%)$ & \\
\hline \multicolumn{7}{|c|}{ Monthly income (in Lebanon \$) } \\
\hline$<300 \$$ & $140(45 \%)$ & $18(50 \%)$ & $36(61 \%)$ & $19(76 \%)$ & $5(100 \%)$ & \\
\hline $300-600 \$$ & 89 (28.6\%) & II (30.6\%) & $16(27.1 \%)$ & $4(16 \%)$ & 0 & \\
\hline $601-1,000 \$$ & $47(15.1 \%)$ & $7(19.4 \%)$ & $5(8.5 \%)$ & $2(8 \%)$ & 0 & \\
\hline $1,00 \mid-1,500 \$$ & $22(7.1 \%)$ & 0 & $\mathrm{I}(1.7 \%)$ & 0 & 0 & 0.157 \\
\hline $\mathrm{I}, 50 \mathrm{I}-2,500 \$$ & $6(1.9 \%)$ & 0 & I (I.7\%) & 0 & 0 & \\
\hline$>2,500 \$$ & $7(2.3 \%)$ & 0 & 0 & 0 & 0 & \\
\hline \multicolumn{7}{|l|}{ Diabetes duration } \\
\hline$<1$ year & $31(10 \%)$ & $2(5.6 \%)$ & $2(3.4 \%)$ & $3(12 \%)$ & 0 & \\
\hline $\mathrm{I}-5$ years & $89(28.6 \%)$ & $5(13.9 \%)$ & $16(27.1 \%)$ & $5(20 \%)$ & I (20\%) & \\
\hline $6-10$ years & $54(17.4 \%)$ & $9(25 \%)$ & $16(27.1 \%)$ & $5(20 \%)$ & $2(40 \%)$ & 0.447 \\
\hline$>10$ years & $137(44.1 \%)$ & $20(55.6 \%)$ & 25 (42.4\%) & $12(48 \%)$ & $2(40 \%)$ & \\
\hline \multicolumn{7}{|l|}{ Sleeping hours } \\
\hline$<6$ hours & $87(28 \%)$ & $16(44.4 \%)$ & $22(37.3 \%)$ & II (44\%) & I (20\%) & \\
\hline 6-7 hours & 144 (46.3\%) & $9(25 \%)$ & $24(40.7 \%)$ & $8(32 \%)$ & I (20\%) & \\
\hline 7-9 hours & $72(23.2 \%)$ & $10(27.8 \%)$ & $12(20.3 \%)$ & $3(12 \%)$ & $3(60 \%)$ & 0.033 \\
\hline$>9$ hours & $8(2.6 \%)$ & $\mathrm{I}(2.8 \%)$ & $\mathrm{I}(\mathrm{I} .7 \%)$ & $3(12 \%)$ & 0 & \\
\hline \multicolumn{7}{|l|}{ Patient relief } \\
\hline Yes & $266(85.5 \%)$ & $30(83.3 \%)$ & $39(66.1 \%)$ & $23(92 \%)$ & $3(60 \%)$ & בחחم \\
\hline No & $45(14.5 \%)$ & $6(16.7 \%)$ & $20(33.9 \%)$ & $2(8 \%)$ & $2(40 \%)$ & 0.002 \\
\hline \multicolumn{7}{|c|}{ Social performance impairment } \\
\hline Yes & 75 (24.1\%) & $14(38.9 \%)$ & $30(50.8 \%)$ & $13(52 \%)$ & $2(40 \%)$ & Oח \\
\hline No & $236(75.9 \%)$ & $22(61.1 \%)$ & 29 (49.2\%) & $12(48 \%)$ & $3(60 \%)$ & 0.00 \\
\hline \multicolumn{7}{|l|}{ Disease perception } \\
\hline Terrified & $48(15.4 \%)$ & $8(22.2 \%)$ & $19(32.2 \%)$ & $10(40 \%)$ & $3(60 \%)$ & \\
\hline Okay with it & $180(57.9 \%)$ & $22(61.1 \%)$ & $36(61 \%)$ & $10(40 \%)$ & I (20\%) & 0.00 \\
\hline Will never be cured & $10(3.2 \%)$ & $3(8.3 \%)$ & 0 & $3(12 \%)$ & I (20\%) & \\
\hline Neutral & $72(23.2 \%)$ & $3(8.3 \%)$ & $3(5.1 \%)$ & $2(8 \%)$ & 0 & \\
\hline Other & $\mathrm{I}(0.3 \%)$ & 0 & $\mathrm{I}(\mathrm{I} .7 \%)$ & 0 & 0 & \\
\hline
\end{tabular}

Notes: aComparative studies were performed using the chi-squared test. 
Table 2 Frequencies of glycemic control according to different levels of depression

\begin{tabular}{|c|c|c|c|c|c|c|}
\hline \multirow[b]{2}{*}{$\begin{array}{l}\text { Glycemic } \\
\text { control }\end{array}$} & \multicolumn{6}{|c|}{ Depression level } \\
\hline & Nondepressed & $\begin{array}{l}\text { Borderline clinical } \\
\text { depression }\end{array}$ & $\begin{array}{l}\text { Moderate } \\
\text { depression }\end{array}$ & $\begin{array}{l}\text { Severe } \\
\text { depression }\end{array}$ & $\begin{array}{l}\text { Extreme } \\
\text { depression }\end{array}$ & $P$-value ${ }^{a}$ \\
\hline $\mathrm{HbAlc} \leq 7 \%$ & 115 (73.7\%) & 14 (8.9\%) & $20(12.8 \%)$ & $6(3.8 \%)$ & $\mathrm{I}(0.6 \%)$ & \multirow{2}{*}{0.843} \\
\hline $\mathrm{HbAlc}>7 \%$ & $140(70 \%)$ & $16(8 \%)$ & $32(16 \%)$ & II (5.5\%) & I (0.5\%) & \\
\hline
\end{tabular}

Note: a Comparative studies were performed using the chi-squared test.

Abbreviation: HbAlc, glycosylated hemoglobin.

Table 3 Frequencies of different levels of depression among different forms of medications, types of oral drugs used, and insulin therapy duration

\begin{tabular}{|c|c|c|c|c|c|c|}
\hline & \multicolumn{6}{|c|}{ Depression level } \\
\hline & Nondepressed & $\begin{array}{l}\text { Borderline } \\
\text { clinical } \\
\text { depression }\end{array}$ & $\begin{array}{l}\text { Moderate } \\
\text { depression }\end{array}$ & $\begin{array}{l}\text { Severe } \\
\text { depression }\end{array}$ & $\begin{array}{l}\text { Extreme } \\
\text { depression }\end{array}$ & $P$-value ${ }^{a}$ \\
\hline \multicolumn{7}{|c|}{ Form of medication } \\
\hline Pills & $229(73.6 \%)$ & $23(63.9 \%)$ & $38(64.4 \%)$ & $16(64 \%)$ & $3(60 \%)$ & \multirow{4}{*}{0.485} \\
\hline Injections & $15(4.8 \%)$ & $4(1 \mathrm{I} .1 \%)$ & $6(10.2 \%)$ & $4(16 \%)$ & I (20\%) & \\
\hline Pills and injections & $63(20.3 \%)$ & $8(22.2 \%)$ & $13(22 \%)$ & $5(20 \%)$ & I (20\%) & \\
\hline None & $4(1.3 \%)$ & $\mathrm{I}(2.8 \%)$ & $2(3.4 \%)$ & $0(0 \%)$ & $0(0 \%)$ & \\
\hline Total & 311 & 36 & 59 & 25 & 5 & \\
\hline \multicolumn{7}{|l|}{ Type of pills } \\
\hline Metformin & $242(72.8 \%)$ & $26(7.8 \%)$ & $42(12.6 \%)$ & $19(5.7 \%)$ & $3(0.9 \%)$ & 0.681 \\
\hline Sulfonylurea & $95(70 \%)$ & $15(11 \%)$ & $15(11 \%)$ & II (8\%) & $0(0 \%)$ & 0.136 \\
\hline DPP4 inhibitors & $47(73.4 \%)$ & $4(6.3 \%)$ & $8(12.5 \%)$ & $2(3.2 \%)$ & $3(4.6 \%)$ & 0.837 \\
\hline SGLT2 inhibitors & $4(44.5 \%)$ & $\mathrm{I}(\mathrm{II} .1 \%)$ & $3(33.3 \%)$ & $0(0 \%)$ & I (II.I\%) & 0.317 \\
\hline GLPI agonists & $4(57.1 \%)$ & $\mathrm{I}(14.2 \%)$ & $2(28.7 \%)$ & $0(0 \%)$ & $0(0 \%)$ & 0.7 \\
\hline \multicolumn{7}{|c|}{ Insulin therapy duration } \\
\hline$<$ l year & $9(11.5 \%)$ & $2(16.7 \%)$ & $0(0 \%)$ & $3(33.3 \%)$ & $0(0 \%)$ & \multirow{3}{*}{0.109} \\
\hline $\mathrm{I}-5$ years & $28(35.9 \%)$ & $3(25 \%)$ & $7(36.8 \%)$ & $3(33.3 \%)$ & $2(100 \%)$ & \\
\hline$>5$ years & $4 \mid(52.6 \%)$ & 7 (58.3\%) & $12(63.2 \%)$ & $3(33.3 \%)$ & $0(0 \%)$ & \\
\hline Yes & 254 (8I.7\%) & 26 (72.2\%) & 37 (62.7\%) & 17 (68\%) & $3(60 \%)$ & 0.011 \\
\hline No & 57 (I8.3\%) & $10(27.8 \%)$ & 22 (37.3\%) & 8 (32\%) & $2(40 \%)$ & 0.011 \\
\hline
\end{tabular}

Note: a Comparative studies were performed using the chi-squared test.

Abbreviations: DDPPIV, dipeptidyl peptidase 4; GLPI, glucagon-like peptide I; SGLT 2, sodium glucose cotransporter 2.

taking insulin with fear of injection being the major cause in 25 patients $(51 \%)$. Other causes were fear of becoming used to it in 12 patients (3\%), financial difficulties in eight patients $(16.32 \%)$, and feeling of embarrassment in four patients $(6.12 \%)$. However, insulin refusal and its causes showed no significant correlation with depression $(P=0.2)$.

Further inquiring revealed that, of the participants receiving treatment, $403(92.4 \%)$ were committed to their treatment, but there was no significant relationship with depression $(P=0.89)$.

In total, 337 patients $(77.3 \%)$ were satisfied with their treatment. There was a significant correlation between treatment satisfaction and depression $(P=0.011)$. Further analysis revealed that those who were not satisfied by the treatment scored higher on BDI with a mean of 16.17 (SD: 9.81) than those who were satisfied who had a mean score of 12.65 (SD: 9.08).

The study showed that $55(12.6 \%)$ participants never visited the physician in the past 6 months. Mean BDI score of these participants was 16.16 (SD: 8.27). Those who visited the physician at least once in the past 6 months had a mean BDI score of 13.06 (SD: 9.45). There was no significant relationship with depression $(P=0.117)$.

In total, $361(82.7 \%)$ were relieved from concerns about the disease by the physician. There was a significant relationship between patient relief and depression $(P=0.002)$. Those who were not relieved by the physician had a higher mean BDI score of 15.87 (SD: 9.57), whereas those who were relieved had a mean of 12.94 (SD: 9.25). 
Table 4 Relation between diabetes complications and depression

\begin{tabular}{|c|c|c|c|c|c|c|}
\hline \multirow[b]{2}{*}{ Diabetic complications } & \multicolumn{6}{|c|}{ Depression levels } \\
\hline & Nondepressed & $\begin{array}{l}\text { Borderline } \\
\text { clinical } \\
\text { depression }\end{array}$ & $\begin{array}{l}\text { Moderate } \\
\text { depression }\end{array}$ & $\begin{array}{l}\text { Severe } \\
\text { depression }\end{array}$ & $\begin{array}{l}\text { Extreme } \\
\text { depression }\end{array}$ & $P$-value \\
\hline $\begin{array}{l}\text { Nephropathy } \\
\text { Yes } \\
\text { No }\end{array}$ & $\begin{array}{l}19(6.1 \%) \\
292(93.9 \%)\end{array}$ & $\begin{array}{l}3(8.3 \%) \\
33(91.7 \%)\end{array}$ & $\begin{array}{l}10(16.9 \%) \\
49(83.1 \%)\end{array}$ & $\begin{array}{l}4(16 \%) \\
21(84 \%)\end{array}$ & $\begin{array}{l}\text { I (20\%) } \\
4(80 \%)\end{array}$ & 0.035 \\
\hline $\begin{array}{l}\text { Retinopathy } \\
\text { Yes } \\
\text { No }\end{array}$ & $\begin{array}{l}121 \text { (38.9\%) } \\
190(61.1 \%)\end{array}$ & $\begin{array}{l}17(47.2 \%) \\
19(52.8 \%)\end{array}$ & $\begin{array}{l}33(55.9 \%) \\
26(44.1 \%)\end{array}$ & $\begin{array}{l}\text { I4 (56\%) } \\
\text { II (44\%) }\end{array}$ & $\begin{array}{l}5(100 \%) \\
0\end{array}$ & 0.005 \\
\hline $\begin{array}{l}\text { Cerebrovascular accidents } \\
\text { Yes } \\
\text { No }\end{array}$ & $\begin{array}{l}5(1.6 \%) \\
306(98.4 \%)\end{array}$ & $\begin{array}{l}2(5.6 \%) \\
34(94.4 \%)\end{array}$ & $\begin{array}{l}4(6.8 \%) \\
55(93.2 \%)\end{array}$ & $\begin{array}{l}2(8 \%) \\
23(92 \%)\end{array}$ & $\begin{array}{l}\text { I (20\%) } \\
4(80 \%)\end{array}$ & 0.017 \\
\hline $\begin{array}{l}\text { Neuropathy } \\
\text { Yes } \\
\text { No }\end{array}$ & $\begin{array}{l}133(42.8 \%) \\
178(57.2 \%)\end{array}$ & $\begin{array}{l}15(41.7 \%) \\
21(58.3 \%)\end{array}$ & $\begin{array}{l}35(56 \%) \\
24(40.7 \%) \\
\end{array}$ & $\begin{array}{l}\text { I4 (56\%) } \\
\text { II (44\%) }\end{array}$ & $\begin{array}{l}2(40 \%) \\
3(60 \%) \\
\end{array}$ & 0.143 \\
\hline $\begin{array}{l}\text { Cardiovascular conditions } \\
\text { Yes } \\
\text { No }\end{array}$ & $\begin{array}{l}30(9.6 \%) \\
281(90.4 \%)\end{array}$ & $\begin{array}{l}6(16.7 \%) \\
30(83.3 \%) \\
\end{array}$ & $\begin{array}{l}5(8.5 \%) \\
54(91.5 \%)\end{array}$ & $\begin{array}{l}5(20 \%) \\
20(80 \%)\end{array}$ & $\begin{array}{l}0 \\
5(100 \%)\end{array}$ & 0.296 \\
\hline $\begin{array}{l}\text { Coronary artery disease } \\
\text { Yes } \\
\text { No }\end{array}$ & $\begin{array}{l}22(7.1 \%) \\
289(92.9 \%)\end{array}$ & $\begin{array}{l}3(8.3 \%) \\
33(91.7 \%)\end{array}$ & $\begin{array}{l}4(6.8 \%) \\
55(93.2 \%)\end{array}$ & $\begin{array}{l}\text { I (4\%) } \\
24(96 \%)\end{array}$ & $\begin{array}{l}0 \\
5(100 \%)\end{array}$ & 0.934 \\
\hline $\begin{array}{l}\text { Skin conditions } \\
\text { Yes } \\
\text { No } \\
\end{array}$ & $\begin{array}{l}31(10 \%) \\
280(90 \%)\end{array}$ & $\begin{array}{l}2(5.6 \%) \\
34(94.4 \%) \\
\end{array}$ & $\begin{array}{l}4(6.8 \%) \\
55(93.2 \%)\end{array}$ & $\begin{array}{l}6(24 \%) \\
19(76 \%)\end{array}$ & $\begin{array}{l}\text { I }(20 \%) \\
4(80 \%) \\
\end{array}$ & 0.116 \\
\hline $\begin{array}{l}\text { Gangrene } \\
\text { Yes } \\
\text { No }\end{array}$ & $\begin{array}{l}2(0.6 \%) \\
309(99.4 \%)\end{array}$ & $\begin{array}{l}0 \\
36(100 \%)\end{array}$ & $\begin{array}{l}0 \\
59(100 \%)\end{array}$ & $\begin{array}{l}0 \\
25(100 \%)\end{array}$ & $\begin{array}{l}0 \\
5(100 \%)\end{array}$ & 0.937 \\
\hline $\begin{array}{l}\text { Hypoglycemic symptoms } \\
<1 / \text { week } \\
\text { I-3/week } \\
>3 / \text { week }\end{array}$ & $\begin{array}{l}71(74.7 \%) \\
17(17.9 \%) \\
7(7.4 \%)\end{array}$ & $\begin{array}{l}12(85.7 \%) \\
2(14.3 \%) \\
0\end{array}$ & $\begin{array}{l}19(67.9 \%) \\
7(25 \%) \\
2(7.1 \%)\end{array}$ & $\begin{array}{l}6(60 \%) \\
1(10 \%) \\
3(30 \%)\end{array}$ & $\begin{array}{l}0 \\
\text { I (50\%) } \\
\text { I (50\%) }\end{array}$ & 0.012 \\
\hline
\end{tabular}

In addition, $64(14.7 \%)$ patients were admitted to the hospital at least once in last 6 months. Hospital admissions were not related to depression $(P=0.401)$.

Regarding patient education about the disease, 377 (86.5\%) got their information from the doctor, $71(16.3 \%)$ learned about their disease from the Internet, $65(14.9 \%)$ got instructions from their family, 34 (7.8\%) watched television programs, $20(4.6 \%)$ attended awareness campaigns, and $41(9.4 \%)$ relied on other sources. However, the only source that was found to be significantly correlated with depression was the Internet $(P=0.049)$. Mean score of depression among Internet users was 10.21 (SD: 7.08).

Diabetic complications were reported in many participants, with the most common being neuropathy in 199 (45.6\%), then retinopathy in 190 (43.6\%), cardiovascular conditions in $46(10.6 \%)$, skin conditions in $44(10 \%)$, nephropathy in $37(18.5 \%)$, coronary artery disease in 30 (6.88\%), cerebrovascular accidents in 14 (3.2\%), and gangrene in 2 patients $(0.5 \%)$. However, retinopathy $(P=0.00)$, nephropathy $(P=0.004)$, and cerebrovascular accidents $(P=0.013)$ were significantly correlated with depression.

Table 4 shows the frequencies of different levels of depression among the diabetic complications and hypoglycemic symptoms.

Figure 1 shows the prevalence of different levels of depression among those bothered from diabetic complications. Those who were severely bothered from diabetic complications scored higher on the depression scale with a mean of 18.84 (SD: 9.5).

Occurrence of hypoglycemic symptoms was significantly correlated with depression $(P=0.016)$. Hypoglycemia was present in 149 (34.2\%) of all participants. From those who had hypoglycemic symptoms, 108 (72.4\%) had less than one episode per week, $28(18.79 \%)$ had between one and three episodes per week, and $13(8.72 \%)$ had more than three times episodes per week. Those who had hypoglycemic symptoms more than three times per week showed the highest mean values of depression of 19.23 (SD: 12.5), while those who 


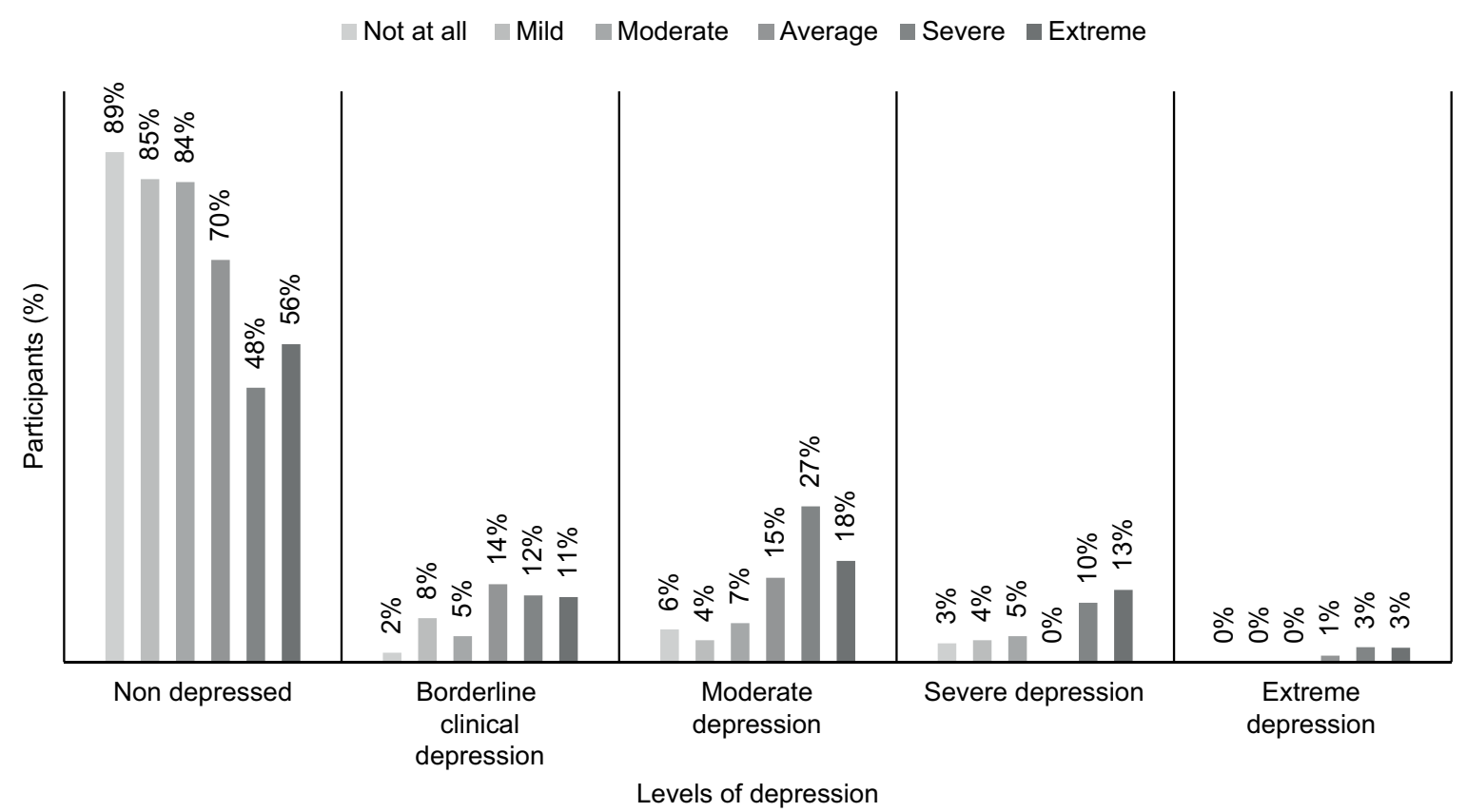

Figure I Percentages of different levels of depression among different levels of bothersome from diabetic complications.

had less than one episode per week had a mean score of 14.66 (SD: 8.24) and those who had one to three episodes per week had a mean score of 15.61 (SD: 11.73).

\section{Impact on lifestyle}

When inquired about their diet, 161 (36.9\%) were annoyed from having restrictions. Diet restrictions, however, had no significant correlation with depression $(P=0.348)$.

Regarding the participants' social performance, 134 (30.7\%) felt that DM impaired their usual activities. It was significantly correlated with depression $(P=0.00)$. Those being socially impaired had a mean score of depression of 16.75 (SD: 9.95), higher than who were not with a mean of 11.98 (SD: 8.7).

Participants were asked about their self-perceived health status, and $12(2.8 \%)$ described it as being the worst, 47 (10.8\%) considered it bad, $54(12.4 \%)$ said it was average, $162(37.2 \%)$ found it to be good, 111 (25.5\%) reported it as being very good, and it was excellent in $50(11.5 \%)$. The overall health status was significantly correlated with depression $(P=0.00)$.

Figure 2 shows the relation between self-estimated overall health status and different levels of depression. Those who perceived their health as worst had the highest mean BDI score of 26.33 (SD: 12.93). Those who perceived it as bad had a mean score of 20.77 (SD: 9.95), those who said it was average had a mean score of 16.07 (SD: 9.56), those who said it was good had a mean score of 13.26 (SD: 7.97), those who described it as very good had a mean score of 11.17 (8.2), and those who said it was excellent had a mean score of 6.3 (SD: 4.95).

As for patient's perception of DM, 88 (20.2\%) felt terrified and had a mean BDI score of 17.09 (SD: 10.69), 249 (57.1\%) were dealing fine with it and had a mean score of 13.5 (SD: 8.45), 17 (3.9\%) felt they would never be cured and had a mean score of 16 (SD: 13.79), 80 (18.3\%) were neutral about DM and had a mean score of 8.6 (SD: 7.23), and $2(0.5 \%)$ could not provide a specific response and had a mean score of 18.5 (SD: 7.77). The perception of DM was significantly related to depression $(P=0.005)$.

\section{Discussion}

The study showed the prevalence of depression to be $28.8 \%$ among our participants with DM in Lebanon. This was found to be lower when compared with other studies conducted in Palestine and Saudi Arabia, where the prevalence of depression was $40.2 \%$ and $49.6 \%$, respectively, ${ }^{12,36}$ while it was found to be higher than other studies done in developed countries, where depression prevalence was $8.3 \%$ in $\mathrm{USA}^{37}$ and $2.6 \%$ in Japan. ${ }^{38}$ Lebanon is a developing country, which explains its position compared with other countries when comparing levels of depression among diabetic patients.

The main purpose of this study was to find whether there exists a relation between glycemic control expressed as $\mathrm{HbAlc}$ levels and depression. However, the analysis of 


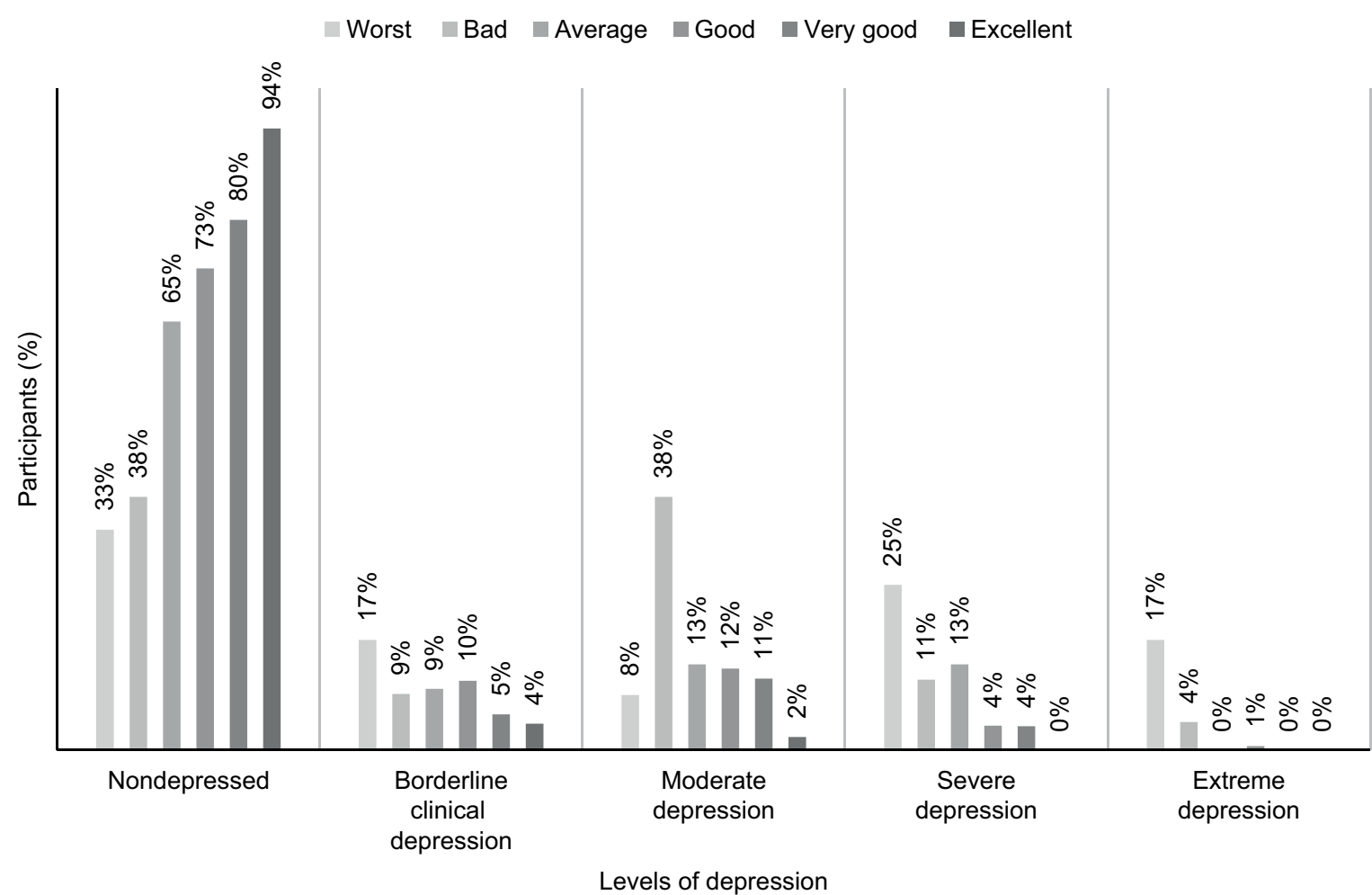

Figure 2 Percentages of different levels of depression among self-perceived health status by the patients.

data showed no significant association between $\mathrm{HbA} 1 \mathrm{c}$ levels and the prevalence of depression $(P=0.843)$. This finding was supported by various research such as those done by Parham et al, ${ }^{39}$ Nejati Safa et al, ${ }^{40}$ Georgiades et al, ${ }^{41}$ and de Groot et $\mathrm{al}^{42}$ who also failed to find any significant relationship between glycemic control and depression among diabetic patients. On the opposite side, a meta-analysis done by Lustman et al among 24 studies indicated the presence of a significant correlation between depression and poor glycemic control. ${ }^{43}$ Other factors, however, played a role in the outcome of depression. These will be further discussed in the upcoming sections.

This study noted that many environmental factors played an important role in affecting the outcome of depression among the participants, such as the place of residence and the occupation. It was found out that participants residing in Beqaa (1.8\%) had more depression than those residing in other areas of Lebanon. In accordance to this result, Probst et al demonstrated that depression levels in residents of rural areas were higher than those of residents of urban areas, as they are more prone to have poor health status, chronic diseases, and poverty, which can affect this outcome ${ }^{44}$ Houle et $\mathrm{al}^{58}$ stated that socioeconomic factors had a major impact on patients' behaviors and depressive symptoms..$^{45}$ The present study is consistent with this statement, as those who were retired $(20.6 \%)$ scored higher.
Other analysis showed that the total amount of sleeping hours the participants had per day were correlated with their BDI score. Most notably, participants who had more than 9 hours of sleep per day (3\%) had higher scores and thus were more depressed. It was well established that there exists a positive relation between depression and excessive daytime sleepiness, expressed as increased daytime sleepiness with increased depression. ${ }^{46}$

While the obtained results failed to prove the existence of a significant relation between glycemic control, expressed as $\mathrm{HbAlc}$ levels, and depression among the participants, the present study found that the regular measurement of $\mathrm{HbAlc}$ was actually implicated in this matter. In total, $10.5 \%$ of the participants did not measure their $\mathrm{HbA} 1 \mathrm{c}$ levels in the past 6 months. These specific individuals had more depression than those who did. Many studies supported the idea that depression is highly associated with $\mathrm{HbA} 1 \mathrm{c}$ levels. The present study, however, found that it was the regular follow-up and not the actual $\mathrm{HbAlc}$ levels that determined depression in the participants.

Bassett et al stated that there was a significant relationship between treatment satisfaction and depression among type 2 DM patients. ${ }^{47}$ In the present study, self-reported treatment satisfaction also had a significant relationship to depression. The more satisfied a patient was with his/her treatment, the less depressed he/she was. 
Concerning physician visits, it was noted that the more the patients visited their physicians, the less they were likely to develop depression. Patients who did not visit their physician at all in the last 6 months (12.6\%) were more depressed compared with those who had at least one visit. In accordance to this result, another study conducted by Delucchi et al stated that the number of clinic visits affected patient's depression $(P=0.03){ }^{48}$

The physician role can affect the outcome of depression in the patient, and when a physician addresses the patient with respect and care, the patient will feel more comfortable and will have a better disease care. ${ }^{49}$

Patient education about DM leads to better glycemic control, ${ }^{50}$ and the present study revealed that the more the patients obtained information about DM, mostly through the Internet, the less depression they had.

Many patients admitted deterioration in their social activities due to DM (30.7\%), which was also related to depression. DM has been associated with multiple cognitive dysfunctions $^{51}$ and even increased the risk of developing dementia in many patients. ${ }^{52}$

Complications related to DM had a significant relation to depression. The present study showed that retinopathy, nephropathy, and strokes were the most important, and it was noted that participants who had a pervious incident of stroke (3.2\%) had higher scores on the BDI scale. Takasaki et al stated that nephropathy is likely associated with increased depression, which is actually decreased following kidney transplant. ${ }^{53}$ Another study stated that not only did retinopathy worsen the presence of depression in diabetic patients but also the depression itself worsened the retinopathy in return. ${ }^{54}$ As for strokes, it was well established that history of type $2 \mathrm{DM}$ and a past cardiovascular accident event were highly predictive of depression and could even lead to dementia. ${ }^{55}$

Hypoglycemia symptoms were among the most notable causes of depression. A study conducted in Japan revealed that hypoglycemic symptoms were strongly associated with severity of depression independent of glycemic control. ${ }^{56}$

Self-perceived health status had a highly significant relation to depression. The better a patient perceived his/her health status, the less depression he/she had. Self-perceived health status is generally considered among the most important indicators of depression. ${ }^{57}$

Illness perception was also strongly correlated with depression. It is thus very essential for hospitals and care centers to manage diabetic patients' disease perception and provide an adequate environment to ensure good information delivery and prevent depression by ensuring good coping behaviors. ${ }^{55}$

\section{Limitations}

There were some limitations encountered in this study to be acknowledged. The participants were selected by convenient sampling, which means that the number of participants from each region of Lebanon was not proportional to the actual population. Participants in Beqaa showed the highest levels of depression; however, the very limited number of participants from this region could have affected this outcome. In addition, diabetic complications were assessed subjectively based only on the patient's medical history and current symptoms, without performing an examination to confirm the diagnosis of these complications. Finally, some participants either had not measured their $\mathrm{HbA} 1 \mathrm{c}$ levels or could not present their test results at the time of interview.

\section{Conclusion}

Depression was found to be prevalent among diabetic patients in Lebanon (28.8\%). However, no association was established between depression and glycemic control. On the other hand, the presence of other disease-related factors was greatly associated with depression, including hypoglycemic symptoms, DM complications especially nephropathy, retinopathy, and stroke, self-reported treatment satisfaction, patient education of the disease, patient-physician relationship, self-perceived health status, and patient's own perception of the disease. Therefore, the early detection of depression in the diabetic patients residing in Lebanon should be an important part of the assessment and management of DM to improve their quality of life and to achieve better therapy outcomes.

\section{Acknowledgments}

The authors thank Mr Akram El Tannir for his help in data analysis. Also, special thanks go to Mr Ali El Tannir for helping in preparation of the manuscript and Mrs Majd Kassem for editing it.

\section{Disclosure}

The authors report no conflicts of interest in this work.

\section{References}

1. Daniele TM, Bruin VM, Oliveira DS, Pompeu CM, Forti AC. Associations among physical activity, comorbidities, depressive symptoms and health-related quality of life in type 2 diabetes. Arq Bras Endocrinol Metabol. 2013;57(1):44-50.

2. International Diabetes Federation. Chapter 3 The global picture: Prevalence and projections. In: IDF Diabetes Atlas. 8th ed. Brussels: International Diabetes Federation; 2017:43-46.

3. International Diabetes Federation. Chapter 4 Diabetes by region: Middle East and North Africa. In: IDF Diabetes Atlas. 8th ed. Brussels: International Diabetes Federation; 2017:72-74. 
4. International Diabetes Federation. Appendix: Country summary table: estimates for 2017. In: IDF Diabetes Atlas. 8th ed. Brussels: International Diabetes Federation; 2017:110-127.

5. Abuyassin B, Laher I. Diabetes epidemic sweeping the Arab world. World J Diabetes. 2016;7(8):165-174.

6. Fowler MJ. Microvascular and Macrovascular Complications of Diabetes. Clinical Diabetes. 2008;26(2):77-82.

7. Thongsai S, Watanabenjasopa S, Youjaiyen M. Depression in patients with type II diabetes: case study at diabetic outpatient clinic, in Samut Prakan. Glob J Health Sci. 2013;6(1):127-134.

8. Rustad JK, Musselman DL, Nemeroff CB. The relationship of depression and diabetes: pathophysiological and treatment implications. Psychoneuroendocrinology. 2011;36(9):1276-1286.

9. American Psychiatric Association. Diagnostic and Statistical Manual of Mental Disorders (DSM-5). 5th ed. Washington, DC: American Psychiatric Association; 2013.

10. El-Asmar K, Chaaya M, Phung KTT, et al. Prevalence of depression and anxiety among older adults in lebanon: preliminary data from Beirut and Mount Lebanon. Alzheimer's Dementia. 2014;10(4):P595.

11. American Diabetes Association. Diagnosis and Classification of Diabetes Mellitus. Diabetes Care. 2012;36(Supp1 1):S62-S69.

12. Sweileh WM, Abu-Hadeed HM, Al-Jabi SW, Zyoud SH. Prevalence of depression among people with type 2 diabetes mellitus: a cross sectional study in Palestine. BMC Public Health. 2014;14:163.

13. American Diabetes Association. Erratum. Glycemic Targets. Sec 6. In Standards of Medical Care in Diabetes-2017. Diabetes Care. 2017;4040(Suppl 1):S48-S56.

14. Stoop CH, Spek VR, Pop VJ, Pouwer F. Disease management for co-morbid depression and anxiety in diabetes mellitus: design of a randomised controlled trial in primary care. BMC Fam Pract. 2011;12(1):139.

15. Sun N, Lou P, Shang Y, et al. Prevalence and determinants of depressive and anxiety symptoms in adults with type 2 diabetes in China: a cross-sectional study. BMJ Open. 2016;6(8):e012540.

16. Crispín-Trebejo B, Robles-Cuadros MC, Bernabé-Ortiz A. Association between depression and glycemic control among type 2 diabetes patients in Lima, Peru. Asia Pac Psychiatry. 2015;7(4):419-426.

17. Carreira M, Anarte MT, Ruiz MS, et al. Depresión en la diabetes mellitus tipo $1 \mathrm{y}$ factores asociados [Depression in type 1 diabetes mellitus and associated factors]. 2010;135(4):151-155. Spanish.

18. Camara A, Baldé NM, Enoru S, Bangoura JS, Sobngwi E, Bonnet F. Prevalence of anxiety and depression among diabetic African patients in Guinea: association with HbA1c levels. Diabetes Metab. 2015;41(1):62-68.

19. Stratton IM, Adler AI, Neil HA, et al. Association of glycaemia with macrovascular and microvascular complications of type 2 diabetes (UKPDS 35): prospective observational study. BMJ. 2000;321(7258):405-412.

20. Nathan DM; DCCT/EDIC Research Group. The diabetes control and complications trial/epidemiology of diabetes interventions and complications study at 30 years: overview. Diabetes Care. 2014;37(1): 9-16.

21. Andreoulakis E, Hyphantis T, Kandylis D, Iacovides A. Depression in diabetes mellitus: a comprehensive review. Hippokratia. 2012;16(3):205-214.

22. Osborn CY, Patel KA, Liu J, et al. Diabetes and co-morbid depression among racially diverse, low-income adults. Ann Behav Med. 2011;41(3):300-309

23. Egede LE, Walker RJ, Bishu K, Dismuke CE. Trends in Costs of Depression in Adults with Diabetes in the United States: Medical Expenditure Panel Survey, 2004-2011. J Gen Intern Med. 2016;31(6):615-622.

24. Wu B, Jin H, Vidyanti I, Lee P-J, Ell K, Wu S. Collaborative Depression Care Among Latino Patients in Diabetes Disease Management, Los Angeles, 2011-2013. Prev Chronic Dis. 2014;11:E148.

25. Berge LI, Riise T, Tell GS, et al. Depression in persons with diabetes by age and antidiabetic treatment: a cross-sectional analysis with data from the Hordaland Health Study. PLoS One. 2015;10(5):e0127161.

26. Katon WJ. The comorbidity of diabetes mellitus and depression. Am J Med. 2008;121(11 Suppl 2):S8-S15.
27. Katon WJ, Young BA, Russo J, et al. Association of depression with increased risk of severe hypoglycemic episodes in patients with diabetes. Ann Fam Med. 2013;11(3):245-250.

28. Bădescu SV, Tătaru C, Kobylinska L, et al. The association between Diabetes mellitus and Depression. J Med Life. 2016;9(2):120-125.

29. Delamater AM, Jacobson AM, Anderson B, et al; Psychosocial Therapies Working Group. Psychosocial therapies in diabetes: report of the Psychosocial Therapies Working Group. Diabetes Care. 2001;24(7):1286-1292.

30. Park M, Reynolds CF 3rd. Depression among older adults with diabetes mellitus. Clin Geriatr Med. 2015;31(1):117-137.

31. Beck AT, Ward CH, Mendelson M, Mock J, Erbaugh J. An inventory for measuring depression. Arch Gen Psychiatry. 1961;4(6):561-571.

32. Beck AT, Steer RA, Carbin MG. Psychometric properties of the Beck Depression Inventory: Twenty-five years of evaluation. Clin Psychol Rev. 1988;8(1):77-100.

33. Abdul Sattar I. [Depression, modern-day disorder-understanding and treatment methods. Kuwait. World of Knowledge Series (239)]. Kuwait: National Council for Culture and Science; 1998. Arabic.

34. Lustman PJ, Clouse RE, Griffith LS, Carney RM, Freedland KE. Screening for depression in diabetes using the Beck Depression Inventory. Psychosom Med. 1997;59(1):24-31.

35. Shaw BF, Vallis TM, McCabe SB. The assessment of the severity and symptom patterns in depression. In: Beckham EE, Leber WR, editors. Handbook of Depression: Treatment, Assessment and Research. Homewood, IL: Dorsey Press; 1985:372-407.

36. El Mahalli AA. Prevalence and Predictors of Depression among Type 2 Diabetes Mellitus Outpatients in Eastern Province, Saudi Arabia. Int J Health Sci (Qassim). 2015;9(2):119-126.

37. Li C, Ford ES, Strine TW, Mokdad AH. Prevalence of depression among U.S. adults with diabetes: findings from the 2006 behavioral risk factor surveillance system. Diabetes Care. 2008;31(1):105-107.

38. Arima H, Miwa M, Kawahara K. The prevalence of co-morbid depression among employees with type 2 diabetes in a Japanese corporation: a descriptive study using an integrated health database. J Med Dent Sci. 2007;54(1):39-48

39. Parham M, Hosseinzadeh F, Hajizadeh J, Norouzinezhad GH. Depressive Symptoms and Glycemic Control in Patients with Type 2 Diabetes: Is There Any Relationship? J Isfahan Med School. 2013; 31(256):1649-1656

40. Nejati Safa A, Larijani B, Shariati B, Amini H, Rezagholizadeh A. Depression, quality of life and glycemic control in patients with diabetes. Iranian J Diabetes Metab. 2007;7(2):195-204.

41. Georgiades A, Zucker N, Friedman KE, et al. Changes in depressive symptoms and glycemic control in diabetes mellitus. Psychosom Med. 2007;69(3):235-241.

42. de Groot M, Jacobson AM, Samson JA, Welch G. Glycemic control and major depression in patients with type 1 and type 2 diabetes mellitus. J Psychosom Res. 1999;46(5):425-435.

43. Lustman PJ, Anderson RJ, Freedland KE, de Groot M, Carney RM, Clouse RE. Depression and poor glycemic control: a meta-analytic review of the literature. Diabetes Care. 2000;23(7):934-942.

44. Probst JC, Laditka SB, Moore CG, Harun N, Powell MP, Baxley EG. Rural-urban differences in depression prevalence: implications for family medicine. Fam Med. 2006;38(9):653-660.

45. Latif E. The impact of retirement on mental health in Canada. J Ment Health Policy Econ. 2013;16(1):35-46.

46. Chellappa SL Araújo JF. Excessive daytime sleepiness in patients with depressive disorder. Rev Bras Psiquiatr. 2006;28(2):126-129.

47. Bassett J, Adelman A, Gabbay R, Aňel-Tiangco RM. Relationship between Depression and Treatment Satisfaction among Patients with Type 2 Diabetes. J Diabetes Metab. 2012;3(7):1000210.

48. Delucchi JC. Relationship of Number of Clinic Visits to Response during Placebo-Controlled Antidepressant Trials in Late Life Depression. J Depression Anxiety. 2015;S1:S1-008.

49. Ritholz MD, Beverly EA, Brooks KM, Abrahamson MJ, Weinger K. Barriers and facilitators to self-care communication during medical appointments in the United States for adults with type 2 diabetes. Chronic Illn. 2014;10(4):303-313. 
50. Kim SH. Educational attainment moderates the associations of diabetes education with health outcomes. Int J Nurs Pract. 2016;22(5):444-450.

51. Moheet A, Mangia S, Seaquist ER. Impact of diabetes on cognitive function and brain structure. Ann NY Acad Sci. 2015;1353(1):60-71.

52. Biessels GJ, Staekenborg S, Brunner E, Brayne C, Scheltens P. Risk of dementia in diabetes mellitus: a systematic review. Lancet Neurol. 2006;5(1):64-74.

53. Takasaki K, Babazono T, Ishizawa K, Miura J, Uchigata Y. Relationship between diabetic nephropathy and depression: a cross-sectional analysis using the Diabetes Study from the Center of Tokyo Women's Medical University (DIACET). BMJ Open Diabetes Res Care. 2016;4(1):e000310.

54. Chen X, Lu L. Depression in Diabetic Retinopathy: A Review and Recommendation for Psychiatric Management. Psychosomatics. 2016;57(5):465-471.
55. Joshi S, Dhungana RR, Subba UK. Illness Perception and Depressive Symptoms among Persons with Type 2 Diabetes Mellitus: An Analytical Cross-Sectional Study in Clinical Settings in Nepal. J Diabetes Res. 2015;2015:908374.

56. Arima H, Miwa M, Kawahara K. The prevalence of co-morbid depression among employees with type 2 diabetes in a Japanese corporation: a descriptive study using an integrated health database. J Med Dent Sci. 2007;54(1):39-48.

57. Wu LR, Parkerson GR Jr, Doraiswamy PM. Health perception, pain, and disability as correlates of anxiety and depression symptoms in primary care patients. J Am Board Fam Pract. 2002;15(3):183-190.

58. Houle J, Lauzier-Jobin F, Beaulieu MD, et al. Socioeconomic status and glycemic control in adult patients with type 2 diabetes: a mediation analysis. BMJ Open Diabetes Res Care. 2016;4(1):e00184.
Diabetes, Metabolic Syndrome and Obesity: Targets and Therapy is an international, peer-reviewed open-access journal committed to the rapid publication of the latest laboratory and clinical findings in the fields of diabetes, metabolic syndrome and obesity research. Original research, review, case reports, hypothesis formation, expert opinion and commentaries are all considered for publication. The manuscript management system is completely online and includes a very quick and fair peer-review system, which is all easy to use. Visit http://www.dovepress.com/testimonials.php to read real quotes from published authors.

Submit your manuscript here: https://www.dovepress.com/diabetes-metabolic-syndrome-and-obesity-targets-and-therapy-journal 